\title{
Structural requirements of $\mathrm{N}$-glycosylation of proteins
}

\author{
Studies with proline peptides as conformational probes
}

\author{
Ernst BAUSE \\ Institut für Biochemie der Universität zu Köln, Zülpicherstrasse 47, D-5000 Köln 1, \\ Federal Republic of Germany
}

(Received 14 June 1982/Accepted 11 October 1982)

\begin{abstract}
Conformational aspects of $\mathrm{N}$-glycosylation have been investigated with a series of proline-containing peptides as molecular probes. The results demonstrate that, depending on the position of the imino acid in the peptide chain, dramatic alterations of glycosylation rates are produced, pointing to a critical contribution of the amino acids framing the 'marker sequence' triplet Asn-Xaa-Thr(Ser) on the formation of a potential sugar-attachment site. No glycosyl transfer at all was detectable to those peptides containing a proline residue either in position $\mathrm{Xaa}$ or in the next position beyond the threonine of the Asn-sequon on the $C$-terminal side, whereas the hexapeptide ProAsn-Gly-Thr-Ala-Val was glycosylated at a high rate. (Emboldened residues denote the 'marker sequence' that is identical in all the peptides; italicized residues distinguish the positions of proline in the various peptides.) Studies with space-filling models reveal that the lack of glycosyl-acceptor capabilities of Ala(Pro)-Asn-Gly-Thr-Pro-Val might be directly related to their inability to adopt and/or stabilize a turn or loop conformation which permits the catalytically essential interaction between the hydroxy amino acid and the asparagine residue within the 'marker sequence' [Bause \& Legler (1981) Biochem.J. 195, 639-644]. This conclusion is supported by circular-dichroism spectroscopic data, which suggest structure-forming potentials in this type of non-acceptor peptides dominating over those that favour the induction of an appropriate sugar-attachment site in the acceptor peptides. The lack of acceptor properties of Tyr-Asn-Pro-Thr-Ser-Val indicates that even small modifications in the 'recognition' pattern are not tolerated by the $N$-glycosyltransferases.
\end{abstract}

The information for the $\mathrm{N}$-glycosylation of particular asparagine residues is supposed to be encoded in the primary structure of the polypeptide chain itself. First statistical evidence for the occurrence of the triplet sequence Asn-Xaa-Thr(Ser) as signal for $N$-glycosylation was presented by Marshall (1967) and Neuberger \& Marshall (1968). Later, this idea of a 'marker sequence' found experimental support, although the significance and possible role of the hydroxy amino acid in the Asn-sequon (Marshall, 1974) still remained obscure (Pless \& Lennarz, 1977; Ronin et al., 1978; Bause, 1979). Recent studies with peptide derivatives that were modified in the side-chain structures of the hydroxy amino acid demonstrated an 'active' participation of this amino acid in the catalytic process of

Abbreviations used: Dol- $P$, dolichyl monophosphate; Dol-PP, dolichyl diphosphate; Xaa, 'unknown' or 'variable' amino acid. transglycosylation (Bause \& Legler, 1981). The realization of this catalytic function requires a hydrogen-bonded interaction between the side chain of asparagine and the hydroxy amino acid within the 'marker sequence' triplet and consequently the adoption of an appropriate conformation of the peptide chain in this region. In fact, Aubert et al. (1976) and Beeley (1977) calculated, by applying the predictive procedure of Chou \& Fasman (1974), a high statistical probability for glycosylated asparagine residues to be located in peptide segments that favour the formation of $\beta$-turns. Circulardichroism spectroscopic studies (Aubert et al., 1981) and model studies with cyclic and linear peptides (Bause et al., 1982) supported this observation.

Continuing the work on conformational aspects of $N$-glycosylation, I report here on a series of proline-containing peptides that are used as molecular and conformational probes. The par- 
ticular structure-forming potential of this imino acid was expected to affect the induction or adoption of 'active' conformations, which itself should be reflected in corresponding alterations of glycosyl-acceptor properties of the various peptide derivatives. The results presented here demonstrate that, depending on the position of the proline residue in the peptide chain, dramatic effects on the glycosylation rates are produced, indicating substantial contributions of the amino acids in the peptide segments adjacent to the 'marker sequence' to the formation of potential sugar attachment sites.

\section{Materials and methods}

\section{Materials}

UDP- $N$-acetyl $\left[{ }^{14} \mathrm{C}\right]$ glucosamine $(323 \mathrm{Ci} / \mathrm{mol})$ was obtained from The Radiochemical Centre. BioBeads S-X1 (chloromethylated) were from Bio-Rad, and $N$-t-butoxycarbonyl-amino acids were from Serva. All other chemicals were purchased from commercial sources in the highest purity available.

\section{Methods}

Preparation of calf liver membranes. Membranes were prepared and isolated essentially as described by Bause et al. (1982). Protein concentration was between 10 and $20 \mathrm{mg} / \mathrm{ml}$, when assayed by the method of Lowry et al. (1951), with bovine serum albumin as standard.

$N$-Glycosyltransferase assay. Glycosyl-acceptor properties of peptides were measured by incubating the microsomal fraction $(100-200 \mu \mathrm{g}$ of membrane protein) with $3 \times 10^{3}$ c.p.m. of Dol-PP- $\left[{ }^{14} \mathrm{C}\right]$ chitobiose in the presence of $0.8 \%$ Triton $\mathrm{X}-100$, $10 \mathrm{~mm}-\mathrm{MnCl}_{2}, 50 \mathrm{~mm}$-Tris/HCl, $\mathrm{pH} 7.2$, and peptide as indicated. The reactions were terminated after $15 \mathrm{~min}$ at room temperature by the addition of $1 \mathrm{ml}$ of methanol and $\left[{ }^{14} \mathrm{C}\right]$ glycopeptides isolated as described previously (Bause et al., 1982).

Synthesis and purification of peptides. Peptides were synthesized by the solid-phase procedure described by Merrifield (1963) and Erickson \& Merrifield (1976). Cleavage from the resin was carried out with $\mathrm{HBr} /$ trifluoroacetic acid in the presence of anisole (methoxybenzene) as bromine scavenger. The peptides were purified by chromatography on Bio-Gel P-4 with $1 \mathrm{M}$-acetic acid as the eluent. Their purity was checked by t.l.c. in butan-1-ol/acetic acid/water (4:1:1, by vol.) and butan-1-ol/acetic acid/water/pyridine $(15: 3: 12: 10$, by vol.). Amino acid analyses of the peptides gave stoichiometric ratios $\pm 5 \%$ for the amino acids according to the expected composition.

Circular dichroism. Circular-dichroism spectra were recorded at room temperature on a Dichrograph Mark III-S (Jobin Yvon, Division d'Instruments S.A., Longjumeau, France) in the region of $185-260 \mathrm{~nm}$ in water or aq. $50 \%(\mathrm{v} / \mathrm{v})$ ethanol respectively. The ellipticity, $[\theta]$, given as degrees $\cdot \mathrm{dmol}{ }^{-1} \cdot \mathrm{cm}^{2}$, refers to mean residue weights of each peptide.

General procedures. Dol-PP- $\left[{ }^{14} \mathrm{C}\right]$ chitobiose was prepared and isolated as described by Bause \& Hettkamp (1979). Radioactivity was measured in a liquid-scintillation counter (Delta 3000, Searle Analytic) using Bray's (1960) reagent as counting fluid. Amino acids were analysed on a Beckman amino acid analyser (model $119 \mathrm{CL}$ ) after hydrolysis of peptides with $5.7 \mathrm{M}-\mathrm{HCl}$ in evacuated tubes for $24 \mathrm{~h}$ at $105^{\circ} \mathrm{C}$.

\section{Results and discussion}

Dol-PP-mediated N-glycosylation of Ala-Asn-GlyThr-Ala-Val and Pro-Asn-Gly-Thr-Ala-Val

The incubation of the hexapeptide Ala-Asn-GlyThr-Ala-Val (peptide I) with a crude microsomal fraction of calf liver in the presence of Dol-

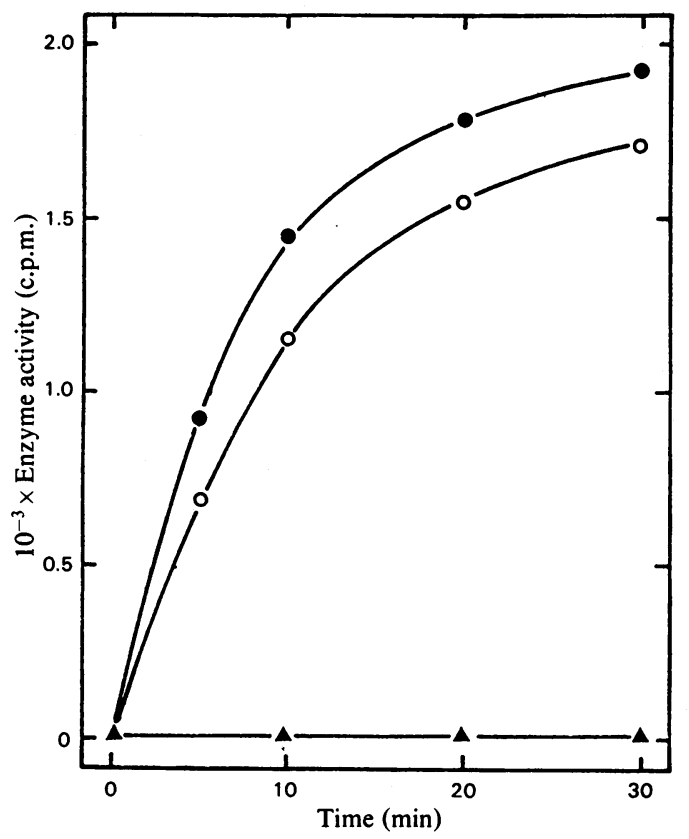

Fig. 1. Time course of glycosyl transfer from Dol$P P-\left[{ }^{14} \mathrm{C}\right]$ chitobiose onto various proline-containing peptides

The crude microsomal fraction from calf liver was incubated wtih 3000 c.p.m. of Dol-PP- $\left[{ }^{14} \mathrm{C}\right]$ chitobiose in the presence of $2 \mathrm{mM}$-Ala-Asn-Gly-ThrAla-Val (O), Pro-Asn-Gly-Thr-Ala-Val (O) or Pro-Asn-Gly-Thr-Pro-Val, Ala-Asn-Gly-Thr-Pro$\mathrm{Val}$ and Tyr-Asn-Pro-Thr-Ser-Val ( $\mathbf{\Delta})$. At given times the reactions were terminated by the addition of $1 \mathrm{ml}$ of methanol and $\left[{ }^{14} \mathrm{C}\right]$ glycopeptides were isolated as described by Bause et al. (1982). 
Table 1. Glycosyl-acceptor properties of synthetic peptides

Glycosylation of peptides was measured as described in the Materials and methods section. Apparent $K_{\mathrm{m}}$ and $V_{\text {max. }}$ values represent mean values $( \pm$ S.D. $)$ of two separate determinations. $K_{1}$ values for non-acceptor peptides are estimated minimum values from inhibition studies (no inhibition with [non-acceptor peptide] = [acceptor peptide]).

Peptide
I
II
III
IV
V

\begin{abstract}
Amino acid sequence
Ala-Asn-Gly-Thr-Ala-Val

Ala-Asn-Gly-Thr-Pro-Val

Pro-Asn-Gly-Thr-Pro-Val

Tyr-Asn-Pro-Thr-Ser-Val
\end{abstract}

$K_{\mathrm{m}}(\mu \mathrm{M})$

$830 \pm 105$

$240 \pm 45$

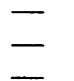

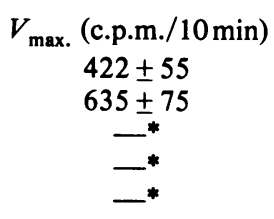

$V_{\max } / K_{\mathrm{m}}$
0.51
2.6
-
-

$K_{\mathrm{i}}(\mathrm{mM})$

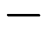

-

$>5$

$>5$

$>5$

* Transfer not detectable.

$P P-\left[{ }^{14} \mathrm{C}\right]$ chitobiose as the glycosyl donor leads to a time-dependent sugar transfer from the glycolipid on to the acceptor peptide (Fig. 1). The observed deviation from linearity is mainly due to peptide depletion caused by endogenous proteinases present in the membrane preparations and consumption of $\left[{ }^{14} \mathrm{C}\right]$ glycolipid donor respectively (E. Bause, unpublished results). Substitution in this peptide of the $N$-terminal alanine residue by proline (peptide II) afforded a derivative with considerably improved glycosyl-acceptor properties, which is shown by the time course (Fig. 1) as well as by the kinetic data given in Table 1. Evidently the particular structure of proline favours, in comparison with alanine, the adoption of an appropriate 'active' conformation, which is documented by the much larger influence of structure on $K_{\mathrm{m}}$ than on $V_{\text {max. }}$.

\section{Non-acceptor peptides}

Under conditions that give rise to a rapid $\mathrm{N}$-glycosylation of peptides I and II, no glycosyl transfer was detectable to those derivatives containing the imino acid either in the next position beyond the threonine of the 'marker sequence' on the $C$-terminal side or in position Xaa within the Asn-Xaa-Thr triplet (Table 1). This lack of acceptor capabilities suggests that the proline residue in these specific positions of the non-acceptor peptides prevents the polypeptide chain adopting a conformation that can be glycosylated. This assumption is supported by the observation that these non-acceptor peptides do not interfere with the glycosylation of potent acceptor peptides. A rough estimation of the kinetic data from inhibition studies points to inhibitory constants for the non-acceptor peptides considerably exceeding $5 \mathrm{~mm}$.

Interestingly, no examples of glycosylated Asnsequons are known in Nature that are followed by proline on the $C$-terminal side, and it appears that the same restrictions also hold for Asn-Pro-Thr(Ser) sequences (Beeley, 1977). This apparent analogy between experimental result and statistical observa- tion suggests that the conclusions derived from the studies in vitro are transferable to the situation in vivo.

Simulation of the three-dimensional structure of a potential sugar-attachment site with space-filling models

Fig. 2 shows a photograph of a space-filling model of the hexapeptide Ala-Asn-Gly-Thr-Ala-Val, which is, in contrast with peptides III, IV and V, (see Table 1), capable of acting as glycosyl acceptor. The peptide backbone is arranged in an $(i+4)$ loop structure that is stabilized by hydrogen-bonding between the $\alpha$-carbonyl of asparagine $(i)$ as the hydrogen-bond acceptor and the $\alpha$-amide function of valine $(i+4)$ as the donor. In this type of secondary structure the hydroxy group of threonine is in hydrogen-bonded contact with the $\beta$-amide function of asparagine (indicated by the arrow), thus fulfilling the structural prerequisite for the catalytic process of transglycosylation to occur (Bause \& Legler, 1981).

In contrast with this, the model of the AlaAsn-Gly-Thr-Pro-Val peptide reveals that, owing to the lack of an $\alpha$-amide proton in the proline molecule, a $\beta$-turn structure $(i+3)$ with asparagine in position $(i)$ and proline in $(i+3)$ cannot be constructed. Also, this particular feature of the imino acid prevents, when being located in this specific position of the peptide chain, stabilization of $(i+4)$ loop, as it turns away the peptide backbone from its plane (Fig. 3). This means that a conformation, comparable with that outlined in Fig. 2 for Ala-Asn-GlyThr-Ala-Val, cannot be induced. This conclusion is supported by the finding that neither Ala-AsnGly-Thr-Pro-Val nor Pro-Asn-Gly-Thr-Pro-Val exert any inhibitory effects on the glycosylation of the other acceptor peptides, indicating that these derivatives are not recognized by the $N$-glycosyltransferases.

Fig. 4 illustrates a possible conformation of the hexapeptide Tyr-Asn-Pro-Thr-Ser-Val with proline 


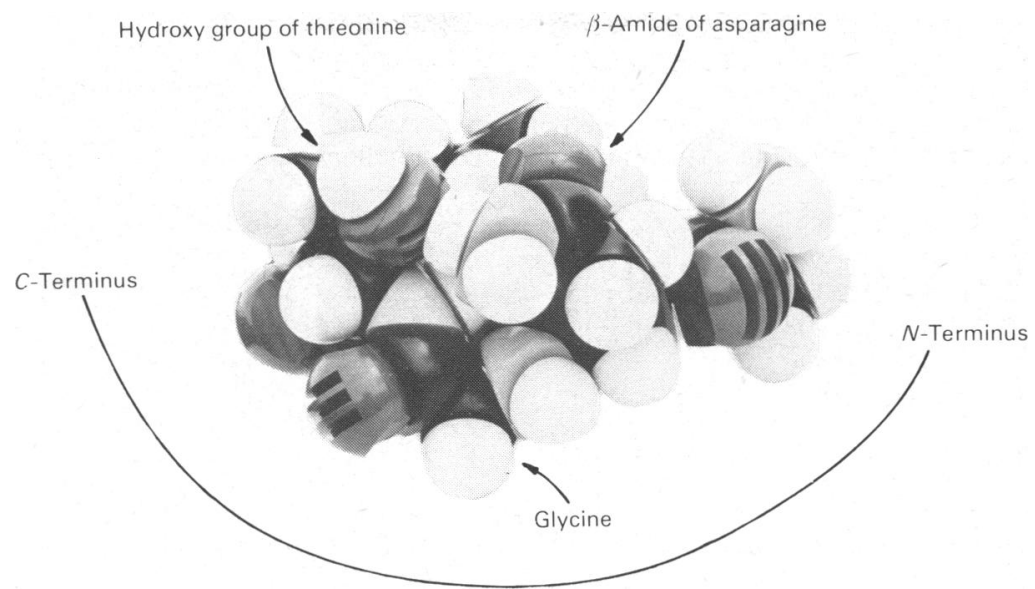

Fig. 2. Space-filling model of the hexapeptide Ala-Asn-Gly-Thr-Ala-Val

The peptide backbone is arranged in an $(i+4)$ loop, which is stabilized by hydrogen-bonding between the $\alpha$-carbonyl group of asparagine ( $i$ ) and the $\alpha$-amide of valine $(i+4)$. The hydroxy group of threonine and the $\beta$-amide group of asparagine (indicated by the arrows) are connected by a hydrogen bond.

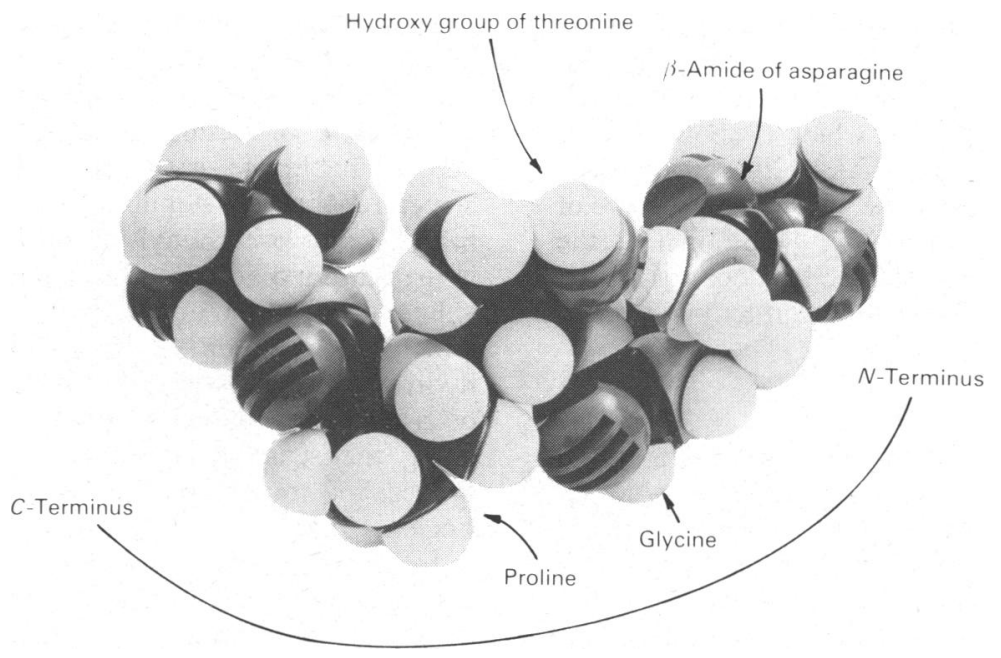

Fig. 3. Space-filling model of the non-acceptor peptide Ala-Asn-Gly-Thr-Pro-Val

For clarity, the $\mathrm{N}$-terminal tetrapeptide segment Ala-Asn-Gly-Thr is shown in the same spatial orientation as the acceptor peptide I in Fig. 2. Owing to the lack of an $\alpha$-proton in the proline molecule, however, this conformation is highly unlikely to be stabilized, e.g. by loop formation. Arrows indicate the position of proline and the side chain of threonine and asparagine respectively.

in position Xaa within the 'marker sequence'. The peptide backbone is arranged here in a turn structure with proline in position $(i+1)$. According to Chou \& Fasman (1974), proline has a high statistical probability to be located in peptide-chain reversals and especially in the $(i+1)$ position of a $\beta$-turn. Although the conformation as shown in Fig. 4 favours the catalytic contact within the Asn-sequon, this peptide cannot be glycosylated under conditions in vitro, apparently contradicting the idea of a sugar-attachment site being located in a loop structure as mentioned above. A comparison of the models in Figs. 2 and 4 shows, however, that the Ala-Asn-Gly-Thr-Ala-Val peptide as glycosyl accep- 


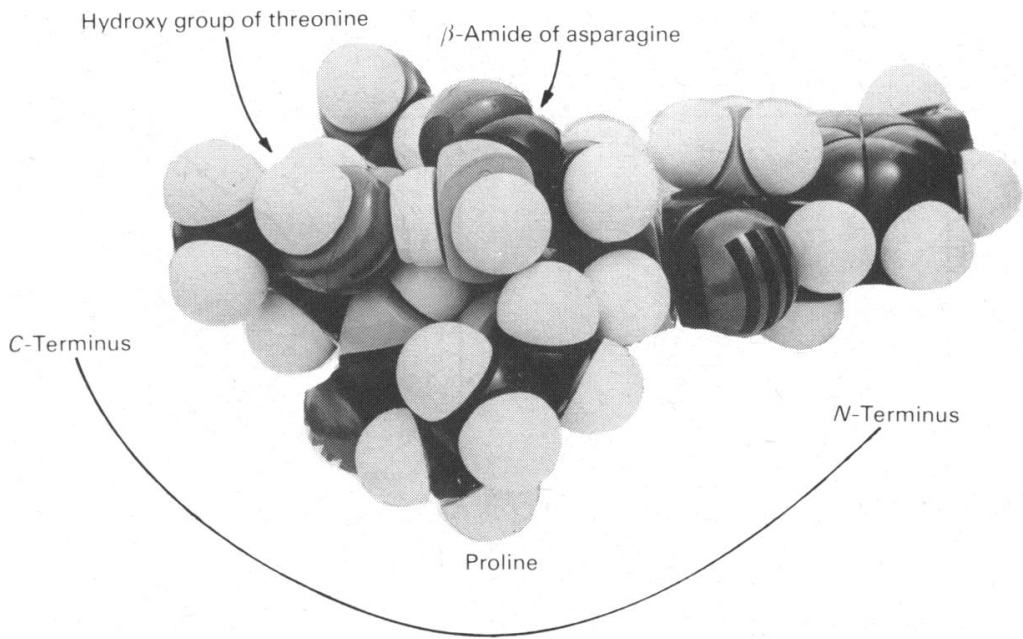

Fig. 4. Model of the non-acceptor peptide Tyr-Asn-Pro-Thr-Ser-Val

The peptide chain is arranged as given in Fig. 2. The position of the proline residue is indicated.

tor and Tyr-Asn-Pro-Thr-Ser-Val as non-acceptor differ in the molecular pattern adjacent to the glycosyl acceptor site by the $\alpha$-amide hydrogen and some steric contributions resulting from the pyrrolidine side chain of proline. It seems likely, and from the energetic point of view conceivable, that these differences in the 'recognition' pattern, might be pronounced enough to alter binding parameters of the corresponding peptides by about two orders of magnitude. Variations in the glycosylation rates of this order of magnitude are not detectable with our enzyme assay in vitro. The critical importance of the $a$-amide proton in this specific position of the Asn-sequon is confirmed by the observation of Ronin et al. (1981) that a heptapeptide containing the triplet sequence Asn- $N$-methylalanine-Thr did not function as glycosyl acceptor, in contrast with its non-methylated analogue.

\section{Circular-dichroism spectroscopic studies of peptides}

The circular-dichroic spectra of the various peptides in water show the expected and typical pattern for unordered conformations, with a strong minimum near $200 \mathrm{~nm}$, as outlined in Fig. 5 for the acceptor peptide Ala-Asn-Gly-Thr-Ala-Val and the non-acceptor Ala-Asn-Gly-Thr-Pro-Val. Increasing the hydrophobicity of the solvent causes only slight shifts in the circular-dichroism curves for the acceptors I and II, indicating still a mixture of unordered conformers present under these conditions. In contrast with that, the circular-dichroism patterns of peptides III and IV changed in that the minimum near $200 \mathrm{~nm}$ disappeared and a new, broad negative band is centred around $210-220 \mathrm{~nm}$. Although an interpretation in terms of specific conformations would be speculation, this result reflects a dominating structure-forming potential differing from those that favour the induction of potential sugar-attachment sites.

\section{Conclusions}

The information obtained with the proline peptides as conformational probes confirm previous findings concerning the possible location of potential sugar-attachment sites in $\beta$-turn or loop structures (Bause et al., 1982). The occurrence of Asn-sequons in this type of secondary structures is favourable for two reasons: (i) $\beta$-turns or loops represent spatial arrangements of the peptide chain which favour the hydrogen-bonded contact between the $\beta$-amide of asparagine and the hydroxy group of the hydroxy amino acid within the 'marker sequence'. Realization of this interaction was taken as a basic criterion for the construction and interpretation of results by the aid of the space-filling models. (ii) $\beta$-turns and loops constitute privileged conformations which guarantee accessibility of the sugar-acceptor sites due to their general location at the surface of proteins.

The glycosylation properties of Pro-Asn-GlyThr-Ala-Val are to be expected, since the proline residue on the $N$-terminal side of the 'marker sequence' triplet does not interfere with the adoption of a loop structure that permits the catalytically essential orientation of the Asn-sequon. In contrast with that, a comparable conformation cannot be constructed for either the Ala-Asn-Gly-Thr-Pro-Val or Pro-Asn-Gly-Thr-Pro-Val peptide, which is in agreement with the lack of acceptor capabilities of these derivatives. Here, the induction of an 'active' 


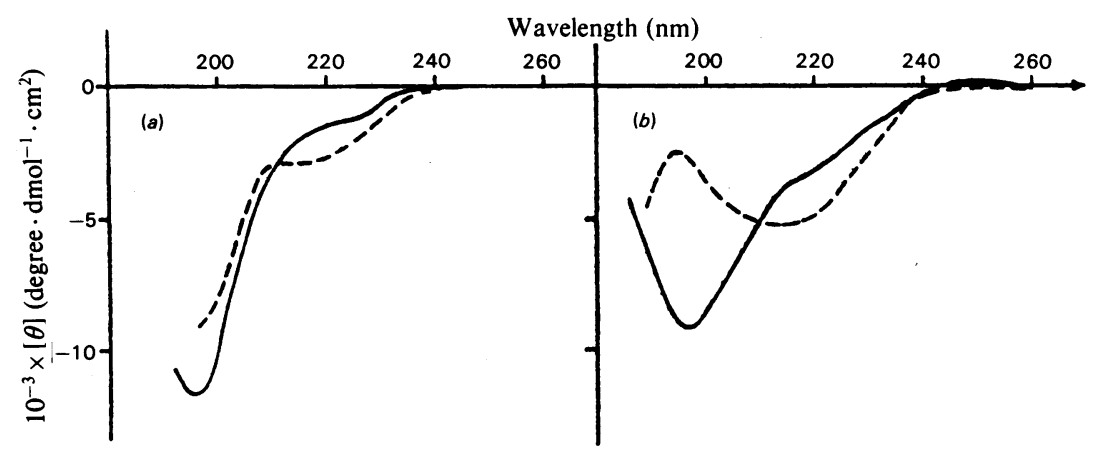

Fig. 5. Circular-dichroism spectra of Ala-Asn-Gly-Thr-Ala-Val (a) and Ala-Asn-Gly-Thr-Pro-Val (b) in water (-) and aq. $50 \%(v / v)$ ethanol (----)

conformation is evidently handicapped by the particular structure-forming potential of the proline residue, which apparently dominates over those factors promoting its formation in acceptor peptides. This interpretation is assisted by the differences in the circular-dichroism spectroscopic pattern of the acceptor and non-acceptor peptides observed in ethanol. Thus the various results demonstrate the critical influence of the nature of the amino acids in the peptide segments adjacent to the 'marker sequence' on the process of $\mathrm{N}$-glycosylation, and it is probable that, under conditions in vivo, corresponding 'short-range' as well as 'long-range' interactions might possess regulating properties in this respect. Similar statements were based on previous systematic studies showing that the glycosylation rates of exogenous acceptor peptides were increasing when the Asn-Xaa-Thr triplet was elongated successively on both the $N$ - and $C$-terminal side by additional amino acids (Bause \& Hettkamp, 1979). The lack of acceptor properties of Tyr-Asn-ProThr-Ser-Val, on the other hand, suggests that, in addition to the aforementioned parameters, even small alterations in the molecular and steric pattern at a sugar-attachment site are recognized, and not tolerated, by the $N$-glycosyltransferases.

I thank Dr. L. Jaenicke and Dr. G. Legler of this Institute for helpful discussions and constructive criticism. I also thank Dr. G. Snatzke (University of Bochum) for kindly performing the circular-dichroism spectroscopic analyses. The work was supported by a grant from the 'Deutsche Forschungsgemeinschaft'.

\section{References}

Aubert, J. P., Biserte, G. \& Loucheux-Lefebvre, M. H. (1976) Arch. Biochem. Biophys. 175, 410-418

Aubert, J. P., Helbecque, N. \& Loucheux-Lefebvre, M. H. (1981) Arch. Biochem. Biophys. 208, 20-29

Bause, E. (1979) FEBS Lett. 103, 296-299

Bause, E. \& Hettkamp, H. (1979) FEBS Lett. 108, 341-344

Bause, E. \& Legler, G. (1981) Biochem. J. 195, 639-644

Bause, E., Hettkamp, H. \& Legler, G. (1982) Biochem. J. 203, 761-768

Beeley, J. G. (1977) Biochem. Biophys. Res. Commun. 76, 1051-1055

Bray, G. A. (1960) Anal. Biochem. 1, 279-285

Chou, P. Y. \& Fasman, G. D. (1974) Biochemistry 13, 222-245

Erickson, B. W. \& Merrifield, R. B. (1976) in The Proteins (Neurath, H. \& Hill, R. L., eds.), vol. 2, pp. 255-562, Academic Press, London and New York

Lowry, P. H., Rosebrough, N. J., Farr, A. L. \& Randall, R. J. (1951) J. Biol. Chem. 193, 265-275

Marshall, R. D. (1967) Abstr. Int. Congr. Biochem. 7th, pp. 573-574

Marshall, R. D. (1974) Biochem. Soc. Symp. 40, 17-26

Merrifield, R. B. (1963) J. Am. Chem. Soc. 85, 21492154

Neuberger, A. \& Marshall, R. D. (1968) in Carbohydrates and their Roles (Schultze, H. W., Cain, R. F. \& Wrotstad, R. W., eds.), p. 115, Avi, Westport, CN

Pless, D. D. \& Lennarz, W. J. (1977) Proc. Natl. Acad. Sci. U.S.A. 74, 134-138

Ronin, C., Bouchilloux, S., Granier, C. \& Van Rietschoten, J. (1978) FEBS Lett. 96, 179-182

Ronin, C., Granier, C., Caseti, C., Bouchilloux, S. \& Van Rietschoten, J. (1981) Eur. J. Biochem. 118, 159-164 